\title{
BMJ Open Protocol for phase I study of pembrolizumab in combination with Bacillus Calmette-Guérin for patients with high-risk non-muscle invasive bladder cancer
}

\author{
Marcus L Jamil, ${ }^{1}$ Mustafa Deebajah, ${ }^{1}$ Akshay Sood, ${ }^{2}$ Kathy Robinson, ${ }^{3}$ \\ Krishna Rao, ${ }^{3}$ Sherjeel Sana, ${ }^{3}$ Shaheen Alanee ${ }^{2}$
}

To cite: Jamil ML, Deebajah M, Sood A, et al. Protocol for phase I study of pembrolizumab in combination with Bacillus Calmette-Guérin for patients with highrisk non-muscle invasive bladder cancer. BMJ Open 2019;9:e028287. doi:10.1136/ bmjopen-2018-028287

\section{- Prepublication history for} this paper is available online. To view these files, please visit the journal online (http://dx.doi. org/10.1136/bmjopen-2018028287).

Received 30 November 2018 Revised 10 May 2019 Accepted 31 May 2019

Check for updates

(C) Author(s) (or their employer(s)) 2019. Re-use permitted under CC BY-NC. No commercial re-use. See rights and permissions. Published by BMJ.

${ }^{1}$ Henry Ford Hospital, Vattikuti Urology Institute Henry Ford Hospital, Detroit, Michigan, USA ${ }^{2}$ Henry Ford Hospital, Vattikuti Urology Institute, Detroit, Michigan, USA

${ }^{3}$ Southern Illinois University School of Medicine, Springfield, Illinois, USA

Correspondence to Dr. Shaheen Alanee; shaheen.alanee@gmail.com

\section{ABSTRACT}

Introduction The initial treatment for high-risk nonmuscle invasive bladder cancer (NMIBC) is endoscopic resection of the tumour followed by BCG therapy. In those who develop recurrence, the standard treatment is radical cystectomy. Despite the advancement in surgical technique and postoperative care, the degree of morbidity associated with radical cystectomy remains high, therefore less invasive treatment modalities are desirable. Therapies targeting the programmed death (PD) pathway have shown promise in urothelial carcinoma. We undertook the current study to determine the safety and efficacy of administering pembrolizumab (a monoclonal antibody targeting the interaction between PD-1 and its ligand) in combination with $\mathrm{BCG}$ in high-risk NMIBC.

Methods This is a single-centre phase I safety and efficacy study of pembrolizumab used in combination with intravesicular BCG treatment for subjects with pathologically documented high-risk NMIBC despite having received two courses of induction therapy or BCG treatment followed by maintenance BCG. Fifteen subjects will be enrolled, patients will receive treatment with $200 \mathrm{mg}$ of pembrolizumab every 21 days, starting 2 weeks from the initial endoscopic resection and continuing for 6 weeks after the final dose of BCG. The primary objective is to determine the safety of administering pembrolizumab at a fixed dose of $200 \mathrm{mg}$ every 3 weeks in conjunction with intravesicular BCG treatment in patients with high-risk NMIBC who have failed previous treatment. Secondary objectives are to determine the 19 weeks and the 3,12 and 24 months post-treatment completion complete response rate with combined pembrolizumab and intravesicular BCG therapy in the aforementioned patients.

Ethics and dissemination The study has been approved by the Institutional Review Board of the Henry Ford Hospital. The results of this study will be published in a peer-reviewed journal and presented at a scientific conference. Trial registration number NCT02324582.

\section{INTRODUCTION}

Bladder cancer is the fifth most common cancer in the USA. ${ }^{1}$ The standard of care for patients who have high-risk non-muscle
Strengths and limitations of this study

- One of the first clinical trials to assess the safety and efficacy of programmed death (PD)-1 inhibitors in combination with BCG for patients with non-muscle invasive high-risk urothelial carcinoma of the bladder after having failed two courses of BCG induction therapy or BCG induction followed by maintenance therapy.

- Study provides extensive follow-up and multiple objective measures assessing the safety and efficacy of PD-1 inhibitors.

- This study is non-randomised and unblinded.

- Long-term enrolment and frequent clinical obligations may result in failure of retention of subjects.

invasive bladder cancer (NMIBC) at presentation is a combination of endoscopic resection and intravesicular BCG instillation. However, only two-thirds of patients respond to BCG, while the remaining one-third experience recurrent disease, which is associated with a poorer prognosis. ${ }^{23}$ Given that the only other accepted treatment for recurrent high-risk NMIBC is a radical cystectomy, a surgery associated with high morbidity, identification of agents active against this disease process is clearly warranted. ${ }^{4}$

The blockade of the immune checkpoint pathway involving programmed death 1 (PD-1) receptor and its ligands, CD274 (PD-L1) and CD273 (PD-L2), has been recently shown to be effective in the treatment of various cancers including urothelial carcinoma (UC). ${ }^{56}$ Previous studies have identified that the expression of PD-L1 has been linked to higher grade disease, and PD-L1 expression has been found to be extremely abundant in BCG-induced bladder granulomata in patients failing BCG treatment. ${ }^{7}$ We 
hypothesise that a combination of pembrolizumab, also known as MK-3475, as it is labelled throughout the study protocol, and BCG following endoscopic treatment of high-risk NMIBC may be effective in improving outcomes of NMIBC after endoscopic treatment in patients unfit or unwilling to be treated with radical cystectomy. The aim of this study is to determine the safety and response of administering pembrolizumab (a monoclonal antibody designed to block the interaction between PD-1 and its ligand) in conjunction with BCG treatment in patients with high-risk NMIBC. In this paper, we report the treatment protocol for the study.

\section{METHODS AND ANALYSIS \\ Rationale}

PD-1 is an inhibitory cell-surface receptor that can be stimulated to be expressed on T cells, B cells, natural killer (NK) T cells, monocytes and dendritic cells (DC). ${ }^{8}$ PD-L1 is widely expressed by haematopoietic, non-haematopoietic and tumour cells, whereas PD-L2 is expressed mainly by DCs and macrophages. ${ }^{9} 10$ Higher expression of PD-L1 in cancers is associated with poor survival as tumours can evade the immune system by stimulating PD-1. ${ }^{11} 12$ PD-L1 expression, a marker for response to anti-PD-1 agents, is observed in 7\% of pTa, $16 \%$ of pT1\% and $45 \%$ of carcinoma in situ (CIS) urothelial tumours. ${ }^{7}$ PD-L1 expression in these tumours is also associated with high-grade disease. In addition, PD-L1 expression is found to be extremely abundant in the BCG-induced transitional epithelium granulomata in patients failing BCG treatment. ${ }^{13}$ Recent pharmacokinetic (PK) studies showing efficacy of anti-PD-1 agents in advanced bladder cancer, advanced melanoma and other cancers support combining these agents with BCG treatment in patients with high-risk superficial bladder cancer to provide these patients with an alternative to radical cystectomy if they fail BCG alone. Previous experience with immunological response supports dosing patients with anti-PD-1 agents 2-3 weeks before BCG treatment, which would allow the bladder to be primed for BCG with the hopes of a greater proportion of complete responses to BCG. ${ }^{13}$ In recent years, pembrolizumab has been investigated, in conjunction with various other chemotherapeutic agents and immunotherapy, in non-metastatic bladder cancer, metastatic bladder cancer and upper tract UC. Based on these data and promising preliminary results, a combination of pembrolizumab with BCG may be effective in preventing recurrence of NMIBC after previously failed treatment.

\section{Trial blinding}

This is an open-label trial; therefore, the sponsor, investigator and subject will know the treatment administered.

\section{Study design}

This is a single-centre phase I safety and efficacy study of pembrolizumab therapy used in combination with bladder infused BCG treatment for subjects, 18 years or

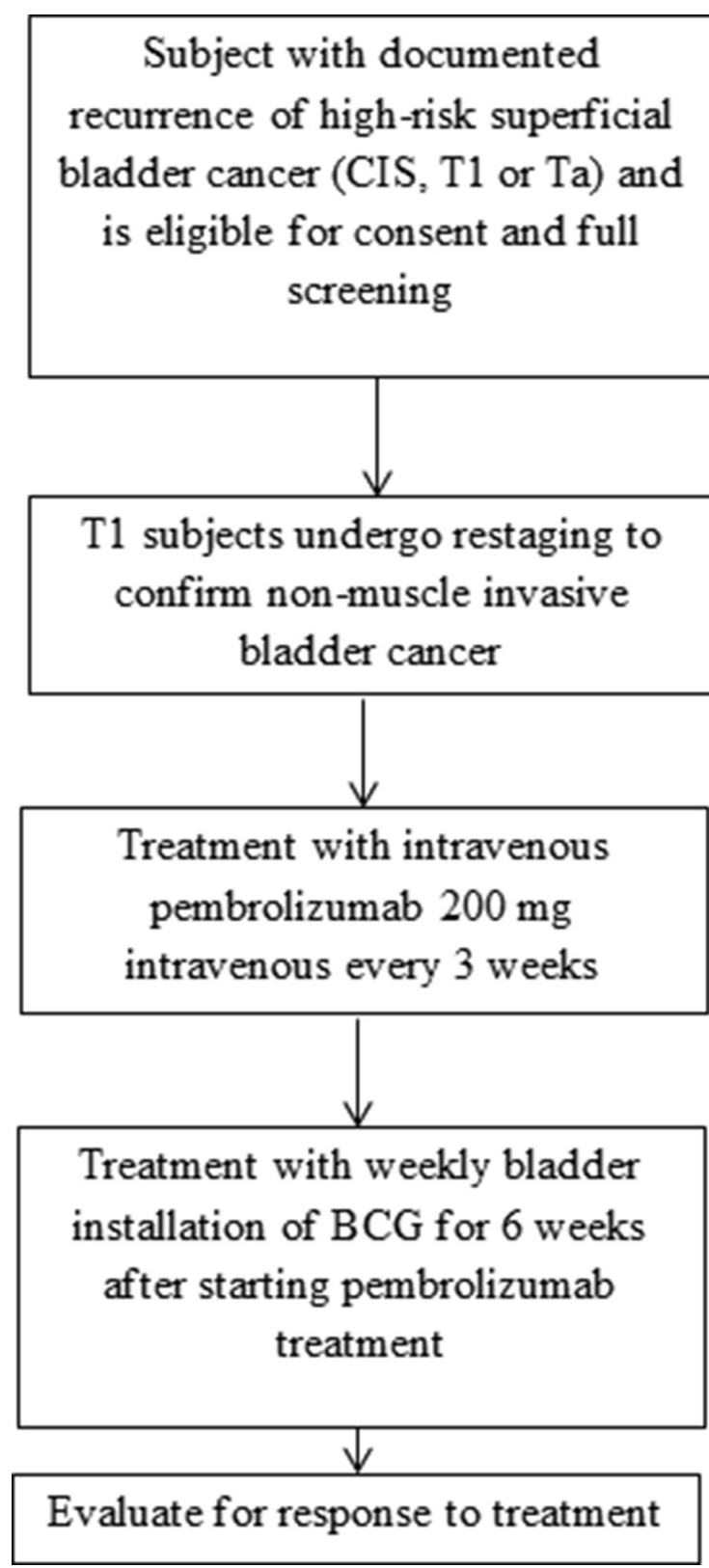

Figure 1 Trial design for phase I study of pembrolizumab in combination with Bacillus Calmette-Guérin for patients with high risk non-muscle invasive bladder cancer. CIS, carcinoma in situ; IFN, interferon.

older, with pathologically documented high-risk NMIBC despite having received two courses of BCG induction therapy or BCG induction followed by maintenance BCG. Subjects will have confirmation of NMIBC. Approximately 20 subjects will be screened to treat 15 eligible subjects. Subjects will be enrolled to a single treatment group of a fixed dose of MK-3475 and BCG (figure 1).

\section{Trial schedule}

Trial schedule (figure 2) summarises the trial procedures to be performed at each visit. It may be necessary to perform these procedures at unscheduled time points if deemed clinically necessary by the investigator. Furthermore, additional evaluations/testing may be deemed 


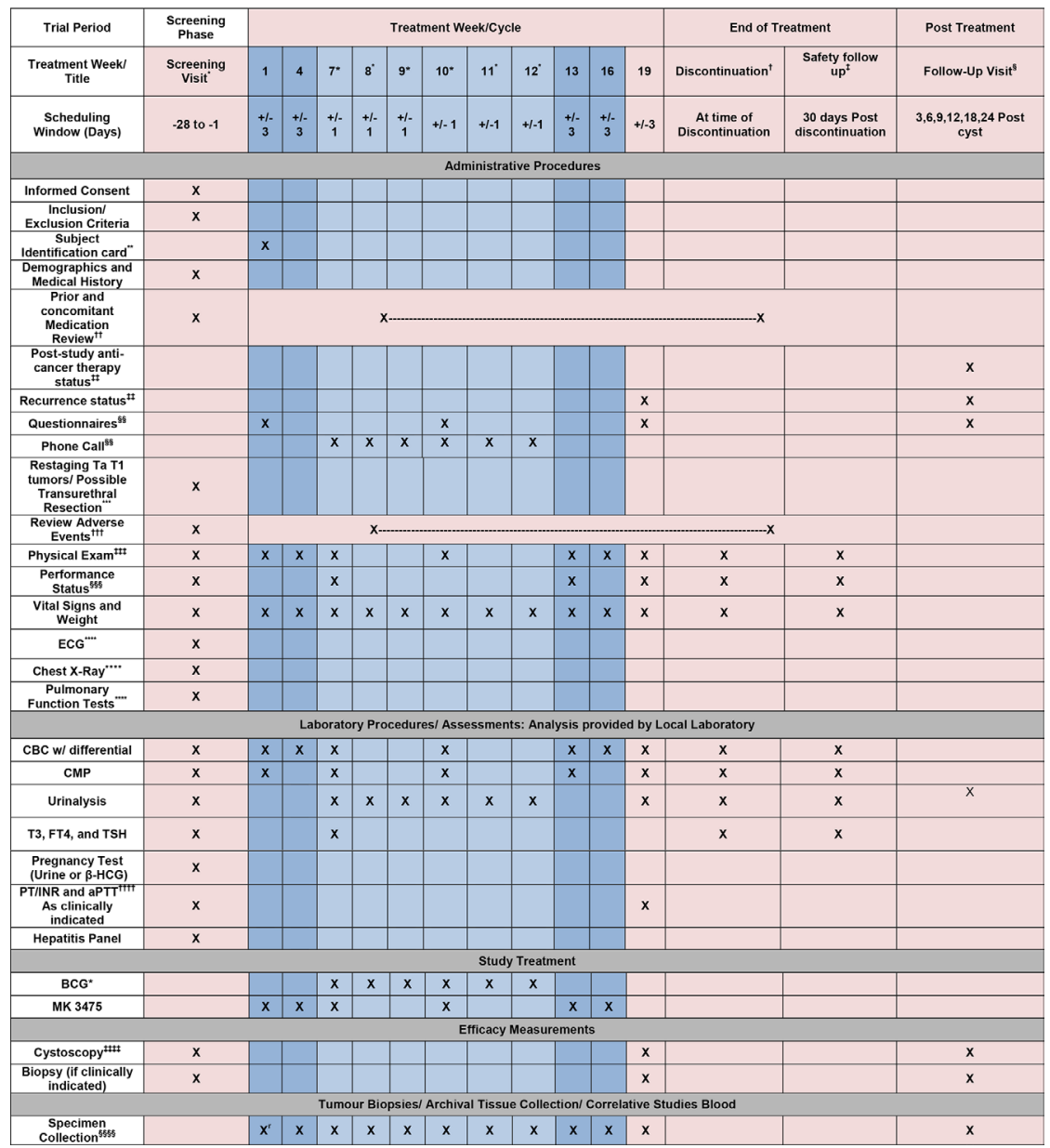

Figure 2 Trial schedule for phase I study of pembrolizumab in combination with Bacillus Calmette-Guérin for patients with high risk non-muscle invasive bladder cancer. 'Screening visit: No study required procedures should be conducted (unless part of routine care) prior to the signing of the informed consent document. Screening visit laboratory procedures may be used for day 1 of cycle 1 if completed within 10 days of day 1 of cycle $1 .{ }^{\dagger}$ Early discontinuation visit: all subjects who discontinue treatment early for any reason should undergo all procedures listed in the flow chart for discontinuation visit. ${ }^{\ddagger}$ If a subject has completed all study treatment, the subject should complete the 30-day safety follow-up visit procedures listed in the flow chart. ${ }^{\S}$ Follow-up visits should occur at approximately 3, 6, 9, 12, 18 and 24 months from week 19 cystoscopy. It is expected that follow-up data will be collected from routine office visits, which generally occur every 3 months in this population. There may be a shift in schedules if the participant has been given maintenance therapy or was found to have a recurrence. Subject will complete a final set of questionnaires at the 3 month visit. Follow-up information may be collected via medical record and document review only. However, patient contact via phone or in person may occur if information is not available via chart review. * Subject identification card may be given at any time after consent process has occurred and prior to treatment on day 1 of cycle $1 .{ }^{\dagger \dagger}$ Prior and concomitant medications will include any medications taken within the 28 days prior to consent and through the safety visit and will include prescribed, over-the-counter and herbal/alternative remedies. ${ }^{\ddagger \ddagger}$ Poststudy treatment anticancer therapy and recurrence status may be collected through follow-up chart review. ${ }^{\S}$ Questionnaires: American Urologic Association Symptoms Index, International prostate symptom score (IPSS) and Quality of Life will be completed prior to any treatment or procedures scheduled for that visit. ${ }^{* \star *}$ Transurethral resections: all TUBR will occur as part of SOC and as clinically indicated. ${ }^{+\dagger \dagger}$ Adverse events will be collected from time of main study informed consent through 30 days post last study drug infusion/treatment. ${ }^{\ddagger \neq \ddagger}$ Physical exam: full physical exams are required at screening and at the 30-day follow-up. All other exams may be symptom directed. ${ }^{\S \S}$ Performance status: Eastern cooperative group (ECOG) performance status scale should be used to measure performance status. ${ }^{* \star *}$ ECG, chest X-ray and pulmonary function tests will be completed prior to day 1 of cycle 1 and will only be repeated as clinically indicated. ${ }^{\dagger+\dagger}$ Prothromin time (PT)/International normalization ratio (INR) and Acticatved prothrombin time (aPTT) will be completed prior to all resections/possibly biopsies as standard of care. All other monitoring of PT/INR and aPTT will only be completed as clinically indicated (ie, if on coumadin). ${ }^{\ddagger \neq \ddagger \ddagger C y s t o s c o p y ~ w i l l ~ b e ~ p e r f o r m e d ~ a s ~}$ standard of care but will be considered measures for efficacy. Biopsy will be performed as clinically indicated. ${ }^{\S \S \S \S}$ Specimen collection/correlative studies: whole blood, serum and urine will be collected at time of consent or may be collected at any time prior to treatment on day 1 of cycle 1. Specimens for correlative study will also occur at each visit through week 19, repeat cystoscopy (12 visits). Saliva will be collected only at baseline. Tumour tissue will only be collected at week 19 biopsy if a biopsy is clinically indicated at the time of cystoscopy. Specimen collection is requested at 3, 6, 9, 12, 18 and 24 months followup visits if subject is being treated locally at the site, although not required. Tissue will only be collected if a biopsy is clinically indicated at time of cystoscopy. 
1. Be willing and able to provide written informed consent for the trial.

2. $\mathrm{Be} \geq 18$ years of age on day of signing informed consent.

3. Have pathologically documented high-grade transitional cell superficial bladder cancer (Ta, T1) at time of restaging, or have pathologically documented high-grade CIS of the bladder at time of initial resection for recurrent/persistent high risk transitional cell superficial bladder cancer.

4. Subject must also meet one of the following criteria: Recurrent/Persistent Disease Despite 2 Induction Intravesical Therapy Courses which includes 2 courses of BCG in a 12-month time period, BCG and mitomycin in a 12 month time period, BCG and gemcitabine in a 12-month time period, treatment with $B C G$ and BCG/interferon combination in a 12-month time period or one BCG treatment in addition to at least one maintenance course of BCG.

5. Have provided tissue from an archival tissue sample or newly obtained core or excisional biopsy of a tumor lesion. Tissue must be obtained from the most recent transurethral bladder resection.

6. Have a performance status of 0-2 on the Eastem Cooperative Oncology Group Performance Scale.

7. Demonstrate adequate organ function.

8. Female subject of childbearing potential should have a negative urine or serum pregnancy test within 72 hours prior to receiving the first dose of study medication. If the urine test is positive or cannot be confimed as negative, a serum pregnancy test will be required.

9. Female subjects of childbearing potential must be willing to use an adequate method of contraception for the course of the study through 120 days after the last dose of study medication.

10. Male subjects of child bearing potential must agree to use an adequate method of contraception starting with the first dose of study therapy through 120 days after the last dose of study therapy.

Figure 3 Subject inclusion criteria for phase I study of pembrolizumab in combination with Bacillus Calmette-Guérin for patients with high risk non-muscle invasive bladder cancer. CIS, carcinoma in situ.

necessary by the sponsor for reasons related to subject safety. In some cases, such evaluation/testing may be potentially sensitive in nature (eg, HIV, hepatitis C, etc), and thus local regulations may require that additional informed consent be obtained from the subject. In these cases, such evaluations/testing will be performed in accordance with those regulations.

Patient selection

Subjects, who meet required inclusion and exclusion criteria are eligible for enrolment into the study (figure 3 and figure 4).

Trial treatment dosage, formulation, preparation and frequency

$B C G$ : the rationale for the use of the indicated dose of BCG is based on Food and Drug Administration (FDA)-approved and commercially provided product. Dosing for the intraluminal treatment of CIS and for the prophylaxis of recurrent papillary tumours consists of one phial of BCG suspended in $50 \mathrm{~mL}$ preservative-free saline. The preparation of the BCG suspension will be completed using aseptic technique and according to FDA-approved labelling and use information. Patients may have treatment with more than one phial to ensure treatment of all tumour sites. BCG treatment will be administered beginning on day 1 of cycle 3 (week 7 ) of pembrolizumab after all procedures/assessments have been completed as detailed on the trial flow chart. BCG treatment will be repeated every 7 days at weeks $8,9,10,11$ and 12. BCG treatment may be administered up to 1 day before or after the scheduled date (at 7 days) due to administrative reasons. All trial treatments will be administered on an outpatient basis and according to institutional standards. BCG dose modification has been shown to reduce efficacy; therefore, no dose modifications will be attempted in this trial. The dose, frequency, route of administration and duration are summarised in figure 5 .

BCG treatment will be withheld for grade 2 haematuria, grade 1 fever and drug-related grade 4 haematological toxicities, and all other non-haematological toxicity 
1. Active or progressive metastatic disease.

2. Is currently participating in or has participated in a study of an investigational agent or using an investigational device within 4 weeks of the first dose of treatment.

3. Has a diagnosis of immunodeficiency or is receiving systemic steroid therapy or any other form of immunosuppressive therapy within 7 days prior to the first dose of trial treatment.

4. Has had a prior monoclonal antibody within 4 weeks before study Day 1 or who has not recovered (ie., $\leq$ grade 1 or at baseline) from adverse events due to agents administered more than 4 weeks earlier.

5. Has had prior systemic chemotherapy, targeted small molecule therapy or radiation therapy for bladder cancer.

6. If subject received major surgery, they must have recovered adequately from the toxicity and/or complications from the intervention prior to starting therapy.

7. Has a known additional malignancy that is progressing or requires active treatment. ${ }^{\dagger}$

8. Active autoimmune disease that has required systemic treatment in the past 2 years. ${ }^{\ddagger}$

9. Has evidence of interstitial lung disease or active, non-infectious pneumonitis.

10. Has an active infection, including a concurrent febrile illness, requiring systemic therapy.

11. Has a history or current evidence of any condition, therapy or laboratory abnormality that might confound the results of the trial, interfere with the subject's participation for the full duration of the trial, or is not in the best interest of the subject to participate, in the opinion of the treating investigator.

12. Has known psychiatric or substance abuse disorders that would interfere with cooperation with the requirements of the trial.

13. Is pregnant or breast feeding, or expecting to conceive or father children within the projected duration of the trial, starting with the prescreening or screening visit through 4 months after the last dose of trial treatment.

14. Has received prior therapy with an anti-PD-1, anti-PD-L1, anti-PD-L2, anti-CD137, or antiCytotoxic T-lymphocyte-associated antigen-4 antibody (including ipilimumab or any other antibody or drug specifically targeting T-cell co-stimulation or checkpoint pathways) including anti-CD40 and anti-OX40 antibodies.

15. Has a known history of Human Immunodeficiency Virus, Hepatitis B (e.g., HBs Ag reactive) or Hepatitis C (e.g., HCV RNA [qualitative] is detected).

16. Has received a live vaccine within 30 days prior to the first dose of trial treatment.

17. Has an active urinary tract infection, gross haematurial or known broken mucosal barrier of the bladder.

Figure 4 Subject exclusion criteria for phase I study of pembrolizumab in combination with Bacillus Calmette-Guérin for patients with high risk non-muscle invasive bladder cancer. "Chest X-ray, CT urogram or MRI and urogram are allowed to ascertain the superficial nature of the disease when indicated, but not required. If urogram protocol is not available or contrast allergy/poor renal function precludes such imaging, then non-contrast CT or MRI of the abdomen/pelvis within 90 days of study entry will suffice. ${ }^{\dagger}$ Exceptions include upper urinary tract transitional cell cracinoma (TCC), basal cell carcinoma of the skin, squamous cell carcinoma of the skin or in situ cervical cancer that has undergone potentially curative therapy. ${ }^{\ddagger}$ Use of diseasemodifying agents, corticosteroids or immunosuppressive drugs. Replacement therapy (eg, thyroxine, insulin or physiologic corticosteroid replacement therapy for adrenal or pituitary insufficiency, etc) is not considered a form of systemic treatment. PD, programmed death.

grade $\geq 3$ adverse effects (AEs) including laboratory abnormalities and severe or life-threatening AEs. Treatment will be postponed until resolution of febrile illness, urinary tract infection or gross haematuria and $\mathrm{AE}$ resolution to grade $<1$. In the event of a grade 1 or 2 immune-related adverse event requiring corticosteroid treatment, BCG treatment may be delayed for up to 2 weeks with the expectation that steroid treatment will be discontinued prior to the next BCG treatment cycle. A delay of longer than 2 weeks will permanently discontinue the patient from BCG treatment within the study or permanent withdrawal of the patient from the study. The choice will be at the discretion of the treating investigator. Steroid treatment for grade 3 or higher immune-mediated events will require discontinuation of BCG treatment and/or withdrawal from the study.

\section{Pembrolizumab}

A fixed dose of $100 \mathrm{mg}$ pembrolizumab will be administered intravenously on day 1 of a 21-day cycle for up to six cycles for the first three subjects. If one subject of the initial three requires the dose of pembrolizumab to be withheld or discontinued, the $100 \mathrm{mg}$ cohort will be expanded to six subjects. If two out of three or three out of six subjects develop toxicity that requires the dose to be withheld or discontinued, the dose will not be escalated 


\begin{tabular}{|c|c|c|c|c|c|}
\hline Drug & Dose/Potency & $\begin{array}{c}\text { Dose } \\
\text { Frequency }\end{array}$ & $\begin{array}{c}\text { Route of } \\
\text { Administration }\end{array}$ & $\begin{array}{c}\text { Regimen/Treatment } \\
\text { Period }\end{array}$ & Use \\
\hline MK-3475 & $100 \mathrm{mg}$ & Every 21 days & $\begin{array}{c}\text { Intravenous } \\
\text { infusion }\end{array}$ & 6 cycles & Experimental \\
\hline MK-3475 & $200 \mathrm{mg}$ & Every 21 days & $\begin{array}{c}\text { Intravenous } \\
\text { infusion }\end{array}$ & 6 cycles & Experimental \\
\hline BCG: & 1 phial & Every 7 days & $\begin{array}{c}\text { Bladder } \\
\text { Instillation }\end{array}$ & 6 cycles & Approved \\
\hline
\end{tabular}

Figure 5 Trial treatment for phase I study of pembrolizumab in combination with Bacillus Calmette-Guérin for patients with high risk non-muscle invasive bladder cancer. *The first three subjects will be dosed at $100 \mathrm{mg}$. If the Data Safety Review Team approves, the dose will be escalated to $200 \mathrm{mg}$ when the fourth subject is enrolled. ${ }^{\dagger}$ The MK-3475 dosing interval may be increased due to toxicity. BCG treatment may be interrupted or delayed as per the instructions for use supplied with BCG. ${ }^{\ddagger} \mathrm{BCG}$ : one phial of BCG suspended in $50 \mathrm{~mL}$ preservative-free saline.

to $200 \mathrm{mg}$ pembrolizumab every 3 weeks. All remaining subjects will be treated at $100 \mathrm{mg}$ pembrolizumab every 3 weeks for six cycles. This is based on multiple clinical trials which have also used pembrolizumab at the aforementioned dose. ${ }^{14}$ An open-label phase I trial (PN001) in subjects with melanoma was conducted to evaluate the safety and clinical activity of single agent pembrolizumab. The dose escalation portion of this trial evaluated three dose levels, 1,3 and $10 \mathrm{mg} / \mathrm{kg}$, administered every 2 weeks in subjects with advanced solid tumours. All three dose levels were well tolerated, and no dose-limiting toxicities were observed. ${ }^{14}$ This first-in-human study of pembrolizumab showed evidence of target engagement and objective evidence of tumour size reduction at all dose levels (1, 3 and $10 \mathrm{mg} / \mathrm{kg}$ every 2 weeks). No maximum tolerated dose was identified. ${ }^{14}$ Selection of $200 \mathrm{mg}$ as the appropriate dose for a switch to fixed dosing is based on simulation results indicating that $200 \mathrm{mg}$ will provide exposures that are reasonably consistent with those obtained with $2 \mathrm{mg} / \mathrm{kg}$ dose and importantly will maintain individual patient exposures within the exposure range established in melanoma as associated with maximal clinical response. A population PK model, which characterised the influence of body weight and other patient covariates on exposure, has been developed using available data from 476 subjects from PN001. The distribution of exposures from the $200 \mathrm{mg}$ fixed dose are predicted to considerably overlap those obtained with the $2 \mathrm{mg} / \mathrm{kg}$ dose, with some tendency for individual values to range slightly higher with the $200 \mathrm{mg}$ fixed dose. The slight increase in PK variability predicted for the fixed dose relative to weight-based dosing is not expected to be clinically important given that the range of individual exposures is well contained within the range of exposures shown in the melanoma studies of 2 and $10 \mathrm{mg} / \mathrm{kg}$ to provide similar efficacy and safety.

Trial treatment will be administered on day 1 of each cycle after all procedures/assessments have been completed as detailed on the trial flow chart. Trial treatment may be administered up to 3 days before or after the scheduled day 1 of each cycle due to administrative reasons. Adverse events (both non-serious and serious) associated with pembrolizumab exposure may represent an immunological aetiology. These adverse events may occur shortly after the first dose or several months after the last dose of treatment. Pembrolizumab must be withheld for drug-related toxicities and severe or life-threatening AEs. Known and theoretical risks of pembrolizumab are documented within the original study protocol and management of said risks and AEs is also noted within the original study protocol.

\section{Objectives}

\section{Primary objective}

To determine the safety of administering pembrolizumab at a fixed dose of $200 \mathrm{mg}$ every 3 weeks in conjunction with intravesicular BCG treatment in patients with highrisk NMIBC who have failed two courses of induction therapy or one course of BCG induction therapy followed by maintenance therapy.

\section{Secondary objective}

To determine the 19 weeks and the 3,12 and 24 months post-treatment completion complete response rate with combined pembrolizumab and intravesicular BCG therapy for patients with non-muscle invasive superficial bladder cancer who have failed two courses of induction therapy or one course of BCG induction therapy followed by maintenance therapy

\section{Exploratory objectives}

Three objectives are to examine pretreatment and post-treatment (MK-3475/BCG) bladder biopsy specimens for PD-1, PD-L1 and CD3; to examine the whole exome sequencing of subjects' germline DNA and formalin-fixed paraffin-embedded tissue and to analyse blood and urine specimens from enrollees for potential development of blood and urine assays for determination of response to treatment.

\section{Adverse effects}

The investigator or qualified designee will assess each subject to evaluate for potential new or worsening AEs as specified in the detailed trial schedule and more frequently, if clinically indicated. Adverse experiences will be graded and recorded throughout the study and during the follow-up period according to the National Cancer 
Institute Common Terminology Criteria for Adverse Events (NCI CTCAE) V.4.0. Toxicities will be characterised in terms of seriousness, causality, toxicity grading and action taken with regard to trial treatment. AEs will be recorded from the time the subject signs the consent through 30 days post the last study treatment. All AEs of unknown aetiology associated with pembrolizumab exposure should be evaluated to determine if it is possibly an event of clinical interest of a potentially immunological aetiology.

All known and theoretical risks and AEs of BCG and pembrolizumab are documented within the original study protocol for reference. Potential AEs and risks are to be monitored for during each mandatory clinical visit. All of the known and theoretical risks and AEs of BCG and pembrolizumab are to be managed by the investigational team and all other necessary support staff. Management of the aforementioned risks and AEs is documented within the original study protocol.

Parameters for determination of patient safety and for trial suspension and discontinuation are noted within the original study protocol. As per the original study protocol, section 5.2.3.3 table 3, all grade 4 toxicities result in permanent discontinuation from the study. Further details and exceptions are noted within the original study protocol. As noted in section 5.8 of the original study protocol, if there are any fatal treatment toxicities or if one or more of the 15 subjects treated experience a grade 4 toxicity, subject accrual will be suspended and all data pertaining to the events will be reviewed by the Study Investigators and Data Safety Review Team to determine if there is the need for any corrective actions. Following review and appropriate action by the investigating team, subject accrual may recommence. If there is a second fatal treatment/morbidity event related to the study treatment or procedures, the study will be terminated. If 4 of 15 subjects experience grade 4 toxicities, the accrual will be suspended and reviewed for added appropriate measures. If five subjects experience grade 4 toxicity, the study will be terminated.

\section{Statistical measures and analysis}

This protocol aims at assessing the activity of one treatment arm, BCG in combination with pembrolizumab in patients with NMIBC. We would like to detect an activity which is at least $25 \%$ greater than that of historical controls (50\%) from case series of BCG-only treatment.

Sample size calculation for this protocol is based on the work by Kahn et al. ${ }^{15}$ Using a one-stage design, 15 patients will be enrolled in this study. Patient-related data will be collected and transferred to an excel spreadsheet for statistical analysis. SAS software V.9.4 will be used for all data analysis. Descriptive statistics will be computed for all study variables. Continuous variables will be described with measures of central tendency (mean, median) and dispersion (range, SD). Categorical variables will be summarised as frequencies and percentages. Some examples include tumour stage (Ta, T1, CIS), age, Eastern cooperative group (ECOG) performance status scale $(0$, $1,2)$, sex and use of tobacco and alcohol.

For primary objective, the proportion of patients with each AE will be reported along with the NCI CTCAE grade of the AE (V.4.0). The Quality of Life Questionnaire, which includes one question with seven categorical responses, will be described with frequencies and percentages. The American Urologic Association Symptoms Index Questionnaire, which includes seven questions each with a numerical score between 0 and 5 as well as a final numerical sum of all responses, will be described with measures of central tendency and dispersion. Due to the questionnaires being given at three time points (pretreatment, mid-treatment and post-treatment), the responses may be analysed to look for longitudinal effects over time for specific variables of interest with repeated measures analysis of variance to evaluate changes and control for individual differences (although this would be very low power due to $n=15$ ).

For the secondary objective, subjects will be assessed at 19 weeks, 3, 12 and 24 months post-treatment completion for complete response. The outcome end point will be complete response status at 19 weeks, 3, 12 and 24 months post-treatment to measure complete response, recorded as yes or no and summarised as proportions and a per cent.

For the exploratory objectives, Pearson's or Spearman's rank correlation coefficient will be used to estimate correlations between expression level change and treatment outcome, as well as the correlations between mutation presence and treatment outcome.

\section{Patient and public involvement}

Patient and the public were not involved in the design, recruitment and conduction of the present study. Results of the study will be disseminated to the study participants on request. Patient advisors were not used in the conduction of this study.

\section{ETHICS, CONFIDENTIALITY AND REGULATORY DETAILS}

The investigator and sponsor of the study will adhere to all applicable data privacy and confidentiality laws and regulations (ie, Health inurance portability and accountability act (HIPAA)). Those applicable include institutional, state and federal law. It is the responsibility of the investigator and sponsor to ensure the subject data as well as sensitive study information is handled according to applicable guidelines and laws. Appropriate authorisation and consent for use, disclosure or transfer of protected health information must be obtained.

Subjects' names will not be recorded on the case report forms (CRF). Only the subject number and subject's initials will be recorded, where permitted. If the subject's name appears on any other document (eg, pathology report), it must be obliterated on the copy of the document as appropriate. Study data stored on a computer will be stored in accordance with local data protection 
laws. The subjects will be informed that representatives of the sponsor or its designee, institutional review board and regulatory authorities may inspect their medical records to verify the information collected, and that all personal information made available for inspection will be handled in strictest confidence and in accordance with local data protection laws.

Qualified staff of the sponsor will monitor the study according to a pre-arranged monitoring plan. Monitoring of the study will include:

- Evaluation of study progress.

- Verification of CRF accuracy and completeness.

- Resolution of inconsistencies in study records.

- Assurance of protocol requirements and investigator's obligations are fulfilled.

- Assurance of compliance with applicable laws and regulations.

Study monitors will periodically review all CRFs and supporting source documentation of participating subjects. The CRFs and supporting documentation must remain up to date and available to the study monitor as arranged. CRF data entry will be verified to correspond with source documentation (laboratory, imaging, pathology reports, etc) and reviewed for completeness. Data discrepancies will be identified and reported to the investigator and site staff.

Protocol deviations will be identified and recorded on a deviation log.

This submission was drafted under the use of the Standard Protocol Items: Recommendations for Interventional Trials reporting guidelines. ${ }^{16}$

\section{DISCUSSION}

This open-label, single-centre, phase I treatment trial intends to investigate the safety and efficacy of pembrolizumab in combination with intravesicular BCG. As previously mentioned, the optimal alternative to management of patients unfit or unwilling to undergo radical cystectomy remains unknown. This prospective clinical trial attempts to provide a more definitive treatment option for NMIBC refractory to BCG. This study uses previously established treatment modalities with available immunotherapeutic agents.

The design of the present study will allow the researchers to discern the safety and efficacy of pembrolizumab and BCG as a viable option for NMIBC. It has been well documented that although radical cystectomy provides definitive cancer control in NMIBC, the associated morbidity and mortality continues to remain high. ${ }^{4} \mathrm{~A}$ non-surgical option following BCG failure is desirable. Various studies have demonstrated promising results in regard to the efficacy of agents such as pembrolizumab and it is presumed that this trend will continue with increased investigation and utilisation. It has been previously demonstrated that the expression of PD-L1 is upregulated in mice models following exposure to BCG, therefore lending to a theoretical synchronous relationship between the two agents and providing further grounds for investigation into this combination therapy. ${ }^{17-19}$ Several investigations are currently underway assessing the potential benefits of pembrolizumab. NCT02808143, a phase I clinical trial, assessing the safety and efficacy of intravesicular pembrolizumab and BCG, in a similar cohort of patients as in the present study, is underway. Notably, the route of administration between the two studies differs, as in our investigation, pembrolizumab is administered intravenously, whereas in NCT02808143, pembrolizumab is administered intravesically. However, despite these differences, it is essential that multiple studies at various institutions be performed to determine the optimal scheduling, dose and route of administration, especially in the early investigations of newer agents.

This potential benefit of pembrolizumab is being assessed beyond patients limited to NMIBC. Of note, pembrolizumab is being investigated in patients who have developed metastatic urothelial cell carcinoma, in combination with other agents or those with muscle-invasive disease. ${ }^{20} \mathrm{~A}$ phase III randomised clinical trial, currently being carried out by Fradet et al, NCT02256436, is seeking to determine the potential benefit of receiving pembrolizumab versus paclitaxel, docetaxel or vinflunine in patients who have metastatic, locally advanced or unresectable urothelial cell carcinoma following recurrence or progression after treatment with platinum-containing chemotherapy. Recent findings from the aforementioned investigation have demonstrated a significant increase in overall survival in those treated with pembrolizumab. It was also noted that the rate of treatment-related AEs was lower in those who received pembrolizumab, $62.0 \%$ than those who received chemotherapy, $90.6 \% .^{2021}$ Other investigations, such as NCT02621151, are currently assessing the combination of concurrent pembrolizumab, chemotherapy and radiation therapy in patients with muscle invasive bladder cancer who are also not deemed surgical candidates. The potential vast utility of pembrolizumab is encouraging.

Ultimately, if the combination of pembrolizumab and BCG is to yield promising results, it would provide patients with an alternative to radical cystectomy and potentially change the standard of care.

Contributors SA, KRo, KRa and SS conceived the idea and the methodology for this clinical trial. The manuscript was drafted and revised by MLJ, MD, AS and SA. All authors read and approved the final manuscript.

Funding This investigator-initiated trial is financially sponsored by Merck \& Co. Competing interests None declared.

Patient consent for publication Not required.

Provenance and peer review Not commissioned; externally peer reviewed.

Open access This is an open access article distributed in accordance with the Creative Commons Attribution Non Commercial (CC BY-NC 4.0) license, which permits others to distribute, remix, adapt, build upon this work non-commercially, and license their derivative works on different terms, provided the original work is properly cited, appropriate credit is given, any changes made indicated, and the use is non-commercial. See: http:// creativecommons.org/licenses/by-nc/4.0/. 


\section{REFERENCES}

1. Jemal A, Murray T, Samuels A, et al. Cancer statistics, 2003. CA Cancer J Clin 2003;53:5-26.

2. Herr HW, Badalament RA, Amato DA, et al. Superficial bladder cancer treated with bacillus Calmette-Guerin: a multivariate analysis of factors affecting tumor progression. J Urol 1989;141:22-8.

3. Hudson MA, Herr HW. Carcinoma in situ of the bladder. $J$ Urol 1995;153(3 Pt 1):564-72.

4. Lavallée LT, Schramm D, Witiuk K, et al. Peri-operative morbidity associated with radical cystectomy in a multicenter database of community and academic hospitals. PLoS One 2014;9:e111281.

5. Brahmer JR, Tykodi SS, Chow LQ, et al. Safety and activity of antiPD-L1 antibody in patients with advanced cancer. N Engl J Med 2012;366:2455-65.

6. Topalian SL, Hodi FS, Brahmer JR, et al. Safety, activity, and immune correlates of anti-PD-1 antibody in cancer. $N$ Engl J Med 2012;366:2443-54.

7. Inman BA, Sebo TJ, Frigola X, et al. PD-L1 (B7-H1) expression by urothelial carcinoma of the bladder and BCG-induced granulomata: associations with localized stage progression. Cancer 2007;109:1499-505.

8. Vegt PD, Witjes JA, Witjes WP, et al. A randomized study of intravesical mitomycin $\mathrm{C}$, bacillus Calmette-Guerin Tice and bacillus Calmette-Guerin RIVM treatment in pTa-pT1 papillary carcinoma and carcinoma in situ of the bladder. J Urol 1995;153(3 Pt 2):929-33.

9. Keir ME, Butte MJ, Freeman GJ, et al. PD-1 and its ligands in tolerance and immunity. Annu Rev Immunol 2008;26:677-704.

10. Zhang P, Su DM, Liang M, et al. Chemopreventive agents induce programmed death-1-ligand 1 (PD-L1) surface expression in breast cancer cells and promote PD-L1-mediated T cell apoptosis. Mol Immunol 2008;45:1470-6.

11. Konishi J, Yamazaki K, Azuma M, et al. B7-H1 expression on non-small cell lung cancer cells and its relationship with tumorinfiltrating lymphocytes and their PD-1 expression. Clin Cancer Res 2004;10:5094-100.
12. Thompson RH, Gillett MD, Cheville JC, et al. Costimulatory B7-H1 in renal cell carcinoma patients: Indicator of tumor aggressiveness and potential therapeutic target. Proc Natl Acad Sci U S A 2004;101:17174-9.

13. De Libero $\mathrm{G}$, Mori L. The T-cell response to lipid antigens of mycobacterium tuberculosis. Front Immunol 2014;5:219.

14. Skinner EC, Goldman B, Sakr WA, et al. SWOG S0353: Phase II trial of intravesical gemcitabine in patients with nonmuscle invasive bladder cancer and recurrence after 2 prior courses of intravesical bacillus Calmette-Guérin. J Urol 2013;190:1200-4.

15. Khan I, Sarker SJ, Hackshaw A. Smaller sample sizes for phase II trials based on exact tests with actual error rates by tradingoff their nominal levels of significance and power. Br J Cancer 2012;107:1801-9.

16. Chan AW, Tetzlaff JM, Altman DG, et al. SPIRIT 2013 statement: defining standard protocol items for clinical trials. Ann Intern Med 2013;158:200-7.

17. Chevalier MF, Schneider AK, Cesson V, et al. Conventional and PDL1-expressing Regulatory T Cells are Enriched During BCG Therapy and may Limit its Efficacy. Eur Urol 2018;74:540-4.

18. Hashizume A, Umemoto S, Yokose T, et al. Enhanced expression of PD-L1 in non-muscle-invasive bladder cancer after treatment with Bacillus Calmette-Guerin. Oncotarget 2018:9:34066-78.

19. Wang Y, Liu J, Yang X, et al. Bacillus Calmette-Guérin and anti-PD-L1 combination therapy boosts immune response against bladder cancer. Onco Targets Ther 2018;11:2891-9.

20. Bellmunt J, Powles T, Vogelzang NJ. A review on the evolution of PD-1/PD-L1 immunotherapy for bladder cancer: The future is now. Cancer Treat Rev 2017;54:58-67.

21. Fradet $\mathrm{Y}$, Bellmunt J, De Wit R, et al. Pembrolizumab (pembro) versus investigator's choice (paclitaxel, docetaxel, or vinflunine) in recurrent, advanced urothelial cancer (UC): 2-year follow-up from the phase 3 KEYNOTE-045 trial. Journal of Clinical Oncology 2018;36(15 suppl):4521. 